\title{
Network based elucidation of drug response: from modulators to targets
}

\author{
Francesco lorio ${ }^{1,3}$, Julio Saez-Rodriguez ${ }^{1}$ and Diego di Bernardo $2,4^{*}$
}

\begin{abstract}
Network-based drug discovery aims at harnessing the power of networks to investigate the mechanism of action of existing drugs, or new molecules, in order to identify innovative therapeutic treatments. In this review, we describe some of the most recent advances in the field of network pharmacology, starting with approaches relying on computational models of transcriptional networks, then moving to protein and signaling network models and concluding with "drug networks". These networks are derived from different sources of experimental data, or literature-based analysis, and provide a complementary view of drug mode of action. Molecular and drug networks are powerful integrated computational and experimental approaches that will likely speed up and improve the drug discovery process, once fully integrated into the academic and industrial drug discovery pipeline.
\end{abstract}

Keywords: Network pharmacology, Drug mode of action, Drug repositioning

\section{Background}

A network is a natural abstraction of a set of objects (nodes) and of the relationships (edges) occurring among them. Nodes and edges in a network may represent heterogenous kinds of relationships, according to the phenomenon being modelled.

Networks have been extensively used to represent regulatory and functional interactions among genes, proteins and metabolites, by mapping experimentally verified, or computationally predicted, interactions as edges between the corresponding nodes [1]. Large-scale genomic, transcriptomic and proteomic experimental data enable the identification of thousands of interactions in a relatively short time, even though their functional meaning is not immediately evident $[1,2]$.

The added value of representing interactions among molecular species as a network stems from the existence of well established theorems and algorithms to identify network level properties, which are not apparent when looking at single interactions $[3,4]$.

Network-based drug discovery and systems pharmacology aim at harnessing the power of networks to investigate

*Correspondence: dibernardo@tigem.it

${ }^{2}$ Telethon Institute of Genetics and Medicine, Naples, Italy

${ }^{4}$ Deptartment of Electrical Engineering and Information Technology,

University of Naples "Federico II", Naples, Italy

Full list of author information is available at the end of the article the impact of small molecules on molecular networks in order to elucidate their mechanism of action and to identify innovative therapeutic treatments [5]. These innovative methodologies can be used to discover: (i) on-target effects, i.e. the intended physical drug-substrate interactions, thus helping in the drug discovery process during lead optimisation (ii) off-target effects, i.e. unforeseen direct physical drug-substrate interactions, and (iii) indirect effects, due to signal propagation after the direct interaction between a drug and its substrates, thus helping in the identification of novel therapeutic opportunities for drug repositioning.

Here, we will review some of the recent advances in the field of network pharmacology, starting with approaches relying on transcriptional networks, then moving to protein and signaling networks and concluding with "drug networks". We will show examples of applications of these methodologies both in drug discovery and in drug repositioning.

\section{Identifying drug mode of action: Transcriptional networks}

Transcriptional (or gene) networks can be broadly defined as a set of nodes representing genes and possibly noncoding RNAs, and a set of edges among genes interacting at the regulatory or functional level (Figure 1). These connections are not necessarily physical interactions, as in the 


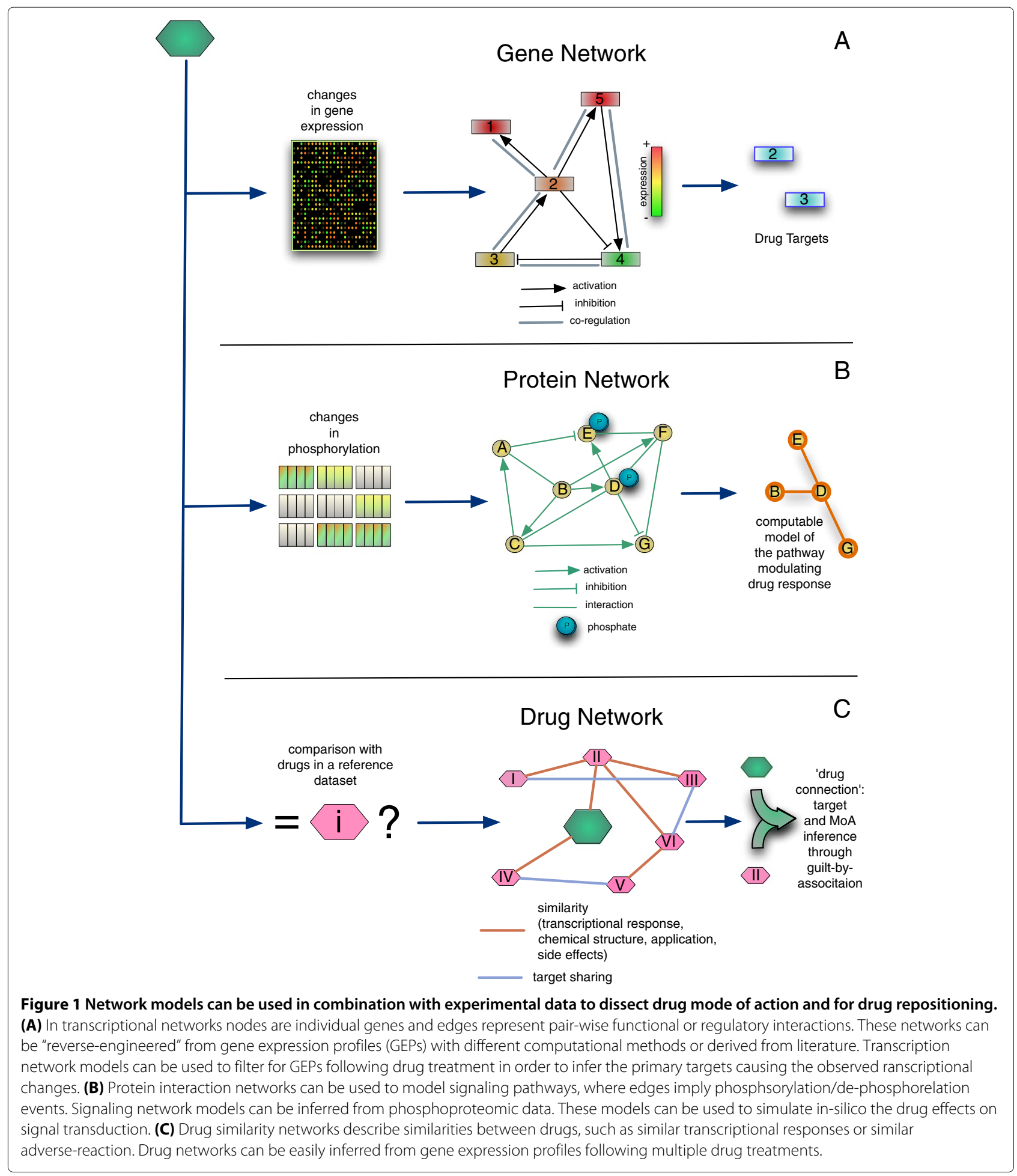

case of protein networks, but can also represent indirect statistical dependencies between genes or ncRNAs [6]. Usually, edges are inferred ("reverse-engineered") from Gene Expression Profiles (GEPs) through computational analysis. Gene expression data from microarrays are typically used for this purpose, but it is likely that Next Generation Sequencing techniques will soon replace them. A gene network can also be compiled using literature-based approaches, without directly using any experimental data. 
The gene network paradigm can be used to represent any one gene in the context of a molecular network that defines the cell behaviour in physiological and pathological conditions [5]. Once a gene network model for a specific cell type or tissue is available, it can be used to "filter" the downstream response of a biological system to a small molecule (or a disease) to identify or confirm its direct molecular targets, as shown in Figure 1. Indeed, changes in the direct targets' activity propagate to other genes through the network, and cause the observed phenotypic response. Thus, gene networks allow to investigate molecular targets of existing drugs, for drug repositiong, as well as to optimise libraries of lead compounds for drug development.

Here, we will review the recent progress made in using gene networks as a tool to elucidate the Mode of Action (MoA) of a compound, that is, the genes and pathways directly modulated by it.

\section{Inference of gene network and drug MoA from steady-state gene expression profiles}

One of the first applications of a gene network reverseengineering approach to identify compound Mode of Action relied on a technique named Network Inference by Regression (NIR) [7].

The authors applied the NIR algorithm in bacteria to reverse-engineer a gene network model consisting of nine genes of the DNA repair SOS pathway; they then used this network to identify direct targets of the chemotherapeutic agent Mitomycin $\mathrm{C}$.

NIR uses multiple linear regression to infer a gene network model from RNA expression changes resulting from a set of steady-state transcriptional perturbations. Specifically, each gene in the SOS pathway was perturbed (i.e. over-expressed) and the transcriptional response of all the genes in the network measured. At the end of the inference procedure, the resulting network was represented by a set of linear differential equations. Each equation described the rate of accumulation of one gene in the network as a linear combination of its regulators, and possibly, of an external perturbation (e.g. a small molecule).

To identify the direct targets of Mitomycin C, the authors first measured RNA expression changes at steadystate resulting from treatment with the compound (i.e. at a single time point following drug administration). The activity of the compound was modeled as a set of unknown external perturbations acting on one, or few genes, in the network. By filtering the differentially expressed genes through the differential equation model of the gene network, the authors were thus able to identify which genes in the network were direct targets of Mitomycin C, and to distinguish them from indirect targets (Figure 1A).
This study represented an interesting proof-ofprinciple, but it was limited in that the inferred gene network was not genome-wide but included only a few selected genes; in addition, the method required each of the gene in the network to be perturbed, by either silencing its expression, or by inducing it, thus making the scaling up of the approach to the genome level very difficult.

In a subsequent study, an extension of the NIR approach, named Mode of action by Network Identification $(\mathrm{MNI})$, was described $[8,9]$. MNI does away with the requirement of having to perturb each of the genes in the network, thus making the approach scalable to the genome level. In MNI, the network is modelled as a system of linear differential equations, as in NIR, and the inference of the network (i.e. the equation parameters) is performed on a compendium of GEPs; the number of GEPs needed for inference, differently from NIR, can be much smaller than the number of genes in the network, thus making the approach easily scalable. Once the network is inferred, it is used to "filter" a single GEP obtained by treating cells with a compound of interest, and to rank genes according to their probability of being the direct targets of the small molecule.

MNI was first applied to a compendium of genomewide GEPs measured in yeast (S. cerevisiae) to identify the molecular targets of anti-fungal compounds [8]. Subsequently, MNI was applied to genome-wide GEPs measured from seven different human cancer cell lines to identify the androgen receptor gene as a genetic mediator of recurrent and metastatic prostate cancer [10].

In the cancer research field, the gene network framework has been applied successfully to identify dysregulated pathways due to oncogenic lesions [11]; a similar approach could be used to identify molecular targets of a small molecule. An algorithm named Interaction Dysregulation Enrichment Analysis (IDEA) has been recently proposed [11]. The method was applied to identify oncogenic lesions in lymphomas. IDEA starts with an "a priori" network model in B cells, assembled by merging different types of experimental and predicted interactions (i.e. protein-protein, co-regulation, etc.), it then uses two sets of GEPs, one comprising GEPs measured in normal B-cell populations and another set measured in B-cell populations from lymphoma patients. By computing differences in co-regulation between genes across the two datasets, using a Mutual Information measure, IDEA was able to identify dysregulated pathways in disease. Although, this method was not applied to identify targets of a small molecule, this in principle could be done. In this case, however, more than one GEP following drug treatment should be available, since Mutual Information requires more than one GEP to be computed. One could compare, for example, drug treated samples to 
untreated samples and predict pathways targeted by the compound.

\section{Inference of gene network and drug MoA from time-course gene expression profiles}

The methods reviewed so far are all based on the availability of several GEPs (in the order of $10^{2}$ for genome-wide applications) measured at a single time point (steadystate) following several different perturbations. These GEPs are then used to infer a gene network model, which in turn is used to filter the drug-induced GEP measured at a single time-point following drug administration.

It is also possible, however, to use a single perturbation, i.e. treatment with the compound of interest, but at multiple time-points (i.e. a time-series) following the drug treatment. From this time-series data, it is then possible to identify the direct molecular targets of the compound and to distinguish them from indirect responses of downstream genes.

An algorithm named Time Series Network Analysis (TSNI), based on this approach, has been proposed by Bansal et al. [12] and applied to infer the targets of the antibiotic Norfloxacin in E. coli. TSNI is similar to NIR and $\mathrm{MNI}$, since it uses a set of linear differential equations to describe the gene network model. Differently from NIR and MNI, however, time-series data are used to identify the equation parameters and detect the direct targets of the perturbation. In this study [12], the authors measured gene expression profiles of nine genes in the SOS pathway at six time-points following treatment of E. coli cells with the antibiotic Norfloxacin. They then applied TSNI to identify the direct mediator of Norfloxacin response among the nine genes.

TSNI was also applied to mammalian cells at the genome-wide level to identify the direct transcriptional targets of the p63 transcription factor in primary murine keratinocytes [13]. The authors measured time-course gene expression profiles at fourteen time-points following inducible activation of the transcription factor using microarrays. TSNI was then applied to the collected GEPs to identify the direct targets of the transcription factor. More recently, TSNI was applied to identify the transcriptional target of Id proteins following inducible deletion of Id genes in murine Neuron Stem Cells [14].

Other methods using time-course gene expression profiles have been developed to reconstruct gene regulatory networks $[15,16]$ and to infer the direct transcriptional targets of a Transcription Factor (TF) $[17,18]$. Some of these methods make use of dynamic Bayesian networks and are based on hidden variables that can capture effects not directly detectable in a gene expression profiling experiment (i.e. genes that have not included in the microarray, levels of regulatory proteins, effects of mRNA and protein degradation) $[15,16]$. To model the effect of the TF on each of the genes and to distinguish direct gene targets from indirect targets of the TF, it is also possible to use simplified model of gene regulation, based on linear differential equations and Gaussian Processes [17,18]. Although the authors of this work did not mention their use to identify drug MoA, in principle these approaches may be applied in a similar fashion to TSNI, if the timecourse GEPs are measured following treatment with a compound of interest.

\section{Literature-derived gene networks for identification of drug MoA}

Literature derived gene and protein networks, obtained by manual curation based on published literature, are a popular way to interpret differentially expressed genes following drug treatment and to identify potential pathways and molecules targeted by the drug. Several tools have been developed to assemble and analyse literature derived biological networks [19]. Usually their interpretation is done by visual inspection, which although useful, cannot be considered as an objective criterion. Different methods have been proposed to solve this problem. Carro et al. applied an algorithm, named Master Regulator Analysis (MRA), which uses a glioblastoma-specific gene network to analyse a "mesenchymal" gene expression signature (MGES), consisting of genes differentially expressed in poor prognosis group of glioma patients in [20]. The algorithm computes the statistical significance of the overlap between the genes connected to each TF in the gene network and the MGES genes, and ranks all the TFs by their likelihood of being direct regulators of the genes in the signature. This algorithm may be used with a literature derived gene network and a list of differentially expressed genes following drug treatment to identify the likely mediators of the drug response.

Along the same lines, Kotelnikova et al presented an algorithm named SubNetwork Enrichment Analysis (SNEA), which uses a similar idea as the MRA algorithm described above: genes differentially expressed in muscle biopsies from Duchenne Muscular Dystrophy patients were mapped to a literature-curated gene network to find master regulators of the differentially expressed genes; a similar approach could be used to elucidate drug mode of action by using differentially expressed genes following drug treatment [21].

\section{Identifying drug mode of action: protein and signaling networks}

Several drugs, such as chemotherapeutic agents, exert their action by affecting the activity of proteins part of the signal transduction machinery. Therefore, the study of signaling networks has potential to enhance our understanding of drug's mode of action. Methods for analyzing protein signaling networks are significantly less mature 
than those for gene regulatory networks, both experimentally and computationally [22]. Nevertheless, some promising approaches have been proposed in the literature and progress is being made at a fast pace. In what follows, we will review some recent applications making use of protein networks to study in silico how drugs operate by perturbing signal transduction pathways (Figure 1B).

The main common feature among the different methods in the literature is the conversion of a protein network into a computational model able to replicate in silico the signaling network function, including its response to perturbations such as drug treatments. These models, in turn, allow mechanistic studies of drug response, with the aim of understanding how interactions between drugs and their targets affect other proteins and cellular components, and thereby cellular phenotype [22].

There are multiple approaches to construct a mathematical model of a signaling pathway with different level of details [23]; more detailed models require more data and knoweldge, thus limiting their scope. We will consider two variants: models that describe signal transduction on the basis of its underlaying biochemistry, which provide detailed mechanistic insight, and others that follow a coarser approach based on describing signaling networks as logic circuits, which provide less detail but cover larger networks.

\section{Biochemical models}

The most common approach to model signal transduction consists of formalising the corresponding biochemical processes and derive from them a dynamic mathematical model (typically as set of differential equations). This feature makes them a natural frame to study drug mode of action, as they can accommodate detailed molecular mechanisms. Furthermore, they can include non-linear effects, allowing to study complex behaviours such as the synergistic combinations of drugs [24,25].

Biochemical models contain a large number of parameters (such as binding constants or total amount of proteins) that are often unknown. These parameters can be found from literature or by training the models on dedicated experimental dataset. Since phosphoproteomic data are relatively difficult to collect, when compared to gene expression data, biochemical models rarely cover more than a couple of pathways and a dozen of proteins [23].

Once a model is set up and the parameters determined, one can analyze it with various techniques. For example, sensitivity analysis provides information on the effect of changes in parameters (such as binding affinities) on the state of model variables (such as the phosphorylation of key protein). This technique can be used to find points of intervention to be targeted with drugs [26] (Figure 1B). Schoeberl et al built a model fo the Receptor Tyrosine
Kinase Family ErbBB and their effect on the key oncogenic pathways MAPK and AKT [27]. Sensitivity analysis revealed a previously unappreciated phenomenon: changes in the amount of ErbB3 receptor affected the phosphorylation of AKT much more than changes in ErbB1 and ErbB2 (which had been the focus of drug development). Subsquently, the authors developed MM121, a human antibody that binds specifically to ErbB3, blocking HRG1-b binding to ErbB3, and inhibits HRG1-band BTC-induced AKT signaling. Follow-up experiments showed effect on mouse tumor xenografts, and clinical trials are currently taking place.

Another use of models is to systematically perform in silico experiments by perturbating the network model with drugs (or combinations of them) to identify adrug combination that may produce a desired outcome (such as to block pro-growth pathways in cancer). Iadeviaia and colleagues developed and calibrated a model of Insulinlike growth factor 1 (IGF1) signaling [28].

Using the model, they predicted, and experimentally validated, that the combined inhibition of the MAPK and PI3K/AKT pathways optimally inhibited the signaling networks and decreased cell viability in a breast cancer cell line.

Biochemical models can also be used to elucidate the molecular mechanisms of action. Hendriks et al. [29] studied a p38 inhibitor postulated to block preferentially the phosphorylation of p38 on one substrate (MK2) versus another (ATF2). Combining detailed biochemical measurements of phospho-MK2 and phospho-ATF2 with a biochemical model, they found a stoichiometric effect in which excess of MK2 could lead to a complex MK2-inhibitor-p38 that would sequester p38, blocking the effect of p38 on ATF2 producing thus the opposite effect to the expected one.

\section{Logic-based models}

Logic-based approaches have become a popular alternative to model signal transduction, since they are based on a simple formalism that can capture cause-effect relationships such as the effects of drug treatments [30-32].

Logic-based models can be used to describe the qualitative behavior of signaling networks even when a limited amount of experimental data is available. The simplest logic-based model is a Boolean model of a signaling pathway, where the phophorylation state of a species can be represented as an ON state (logical value of 1) to indicate the activation of the protein (kinase/phosphatase/transcription factor); analogously, an inactive protein can be represented by the OFF state (logical value of 0 ). The Boolean model of a signaling pathway can thus be thought of as a network where nodes represent proteins, which can either be on or off, and edges 
(or 'hyperedges', representing multiple edges between the same pair of nodes) represent logical operations between proteins (such as logic ANDs and ORs).

Because of their scalability, logic models can be trained against high-throuhgput experimental data, and thus generate large-scale cell-specific models. Differences between healthy versus diseased cell models point at potential targets for therapies: blocking an interaction that is only functional in a disease cell should have a disease-specific effect withouth affecting healthy cells. In a recent study [33], the authors trained a general protein signaling network to phosphoproteomic data generated in primary hepatocytes and four cell lines representing different stages of the development of hepatocellular carcinoma. A comparison of the resulting models revealed key differences between normal and transformed hepatocytes in three different pathways.

Furthermore, logic models can be used to understand drug's mode of action, and in particular provide largescale insights accross many different pathways, which cannot be modelled with biochemical models. In the study discussed above, the authors observed an effect of TPCA1 (an IKK-inhibitor) on JAK/STAT signaling that could not be reconciled with their model based on prior knowledge of the involved pathways. Follow-up experiments including another IKK inhibitor (BMS345541) showed that the effect was specific to TPCA-1, and thus based on an off-target effect rather than an unknown crostalk between Ikb/NFkb and JAK2/STAT pathways [33]. In a related study [34], a similar procedure was used to generate models specific to certain drugs. By comparing the changes in the functional wiring with and without drugs, the authors found beside obvious effects (e.g., EGFR inhibitors blocks the EGFR pathway) not-reported alterations of signal transduction due to drug promiscuity (e.g. EGFR inhibitor Gefitinib inhibits IL1a-mediated activation of JNK activation).

Logic models can be used to systematically explore the properties of large networks to identify therapeutical targets. Saadatpour et al. performed a perturbation analysis of a model of signalling in T-cells, that lead to the identification of 19 potential targets for large granular lymphocyte leukemia, a diseased that exhibits an abnormal increase in the amount of T-cells [35].

In another application of logic modeling, Sahin et al. built a literature-derived logic model of ERBB receptorregulated G1/S transition [36]. They used their model to investigate a chemotherapeutic resistant cell line (specifically, breast cancer cell with de novo tratuzumab resistance) and to find targets whose knockdown would increase drug senstivity. Furthermore, with their model the authors proposed (and experimentally validated invitro) c-MYC as a novel therapeutic target in both resistant and sensitive to trastuzumab breast cancer cell lines.

\section{Identifying drug mode of action: drug networks}

The immediate interpretability of networks and the solid algorithmic background of graph-theory have been recently exploited in computational drug discovery, where "drug-networks" describing different kind of relationships among drugs, diseases, and molecular targets have been successfully developed. In what follows we review some of the most promising results achieved so far.

\section{Gene-Expression based networks for drug-classification and -repositioning}

Differently from the networks described in the previous sections, a number of approaches has been developed on the idea of inferring drug-drug and/or drug-disease similarity networks from gene expression data. In drug networks, each node represents a drug (or disease) and each edge a significant similarity or "anti-similarity" between nodes. In order to build these networks, a number of issues must be solved. Most of them are linked to the integration of data coming from different experimental settings, which are often difficult to merge together without introducing biases, due to batch effects and individualexperiment specificities [37].

As shown in Figure $1 \mathrm{C}$, the common hypothesis underlying these gene expression based methods is that every biological state can be described through a proper gene signature, which can be defined as a set of genes combined with a specific pattern of expression. By making use of "partial" or "genome-wide" metrics, similarity in gene expression signatures (or anti-similarity, when the sign of expression changes are reverted), summarising drug transcriptional responses and/or disease phenotypes, can be used to build drug-drug (DD) or drug-disease (Dd) networks. These networks, in turn, can be queried to identify the potential therapeutic effects and off-targets of a new molecule, by analysing the drugs connected to it in the network ("guilt-by-association" analysis), or to identify new applications for already approved drugs (i.e. "drug repositioning"). A comprehensive review on these two classes of methods has been recently published [38].

The usefulness of a Drug-drug network (Figure 1C) is based on the hypothesys that if gene signatures summarising the effect of drugs are significantly similar to each other, and hence the drugs are connected in the drugdrug network, then those drugs will likely share a common MoA [39].

Drug-disease networks rely on the following assumption: if the gene signature summarizing the effect of a drug is significantly anti-similar with the gene signature characterizing a disease state, then it is reasonable to hypothesise that the drug could "revert" the disease signature, hence the disease phenotype [40]

Based on these assumptions a significant number of new clinical applications for already existing drugs have been 
identified by querying drug-disease networks [41-44] or drug-drug networks $[45,46]$.

To establish edges between drugs or between a drug and a disease in most of the approaches proposed so far, the authors used as reference dataset the Connectivity Map (cMap) [47]: the first large installment of gene expression profiles following drug treatment on five human cancer cell lines with more than 1,000 different bioactive small molecules. In this work, the authors also proposed a non parametric method based on the Kolmogorov-Smirnoff statistic [48] to find connections between a predefined gene signature and the gene expression profiles in the cMap [49].

Moving along these lines, $\mathrm{Hu}$ and Agarwal [50] inferred a drug-disease networks in which two nodes (that can represent both drugs and diseases) were connected if the corresponding signatures were significantly similar or significantly anti-similar. In order to compose disease signatures to be integrated with the cMap drug signatures, the authors mined the Gene Expression Omnibus (GEO) repository [51]. By analyzing the anti-similarities between drugs and disease in the network, they were able to predict new clinical applications for existing drugs, such as the potential efficacy of some antimalaria drugs in treating Chron's disease, and that of other established drugs for Huntington disease.

While derived from similar principles, Iorio et al first generated a drug-drug network from the cMap dataset using a novel "drug distance metric" able to score the similarity between genome-wide GEPs following drug treatment [45]. The network was then analysed with graph-theoretic tools to identify "communities" of drugs consisting of groups of densely interconnected nodes (i.e. drugs). The authors found that these communities were indeed enriched for drugs sharing a common MoA and therapeutic application. The network was then used to classify both known and novel HSP90 inhibitors and CKD2 inhibitors by integrating them in the network and analysing the MoAs enriched in the surrounding communities. In addition, by analysing the neighborhood of a "seed compound" with a desired MoA they were able to predict and experimentally validate that Fasudil, a known ROCK inhibitor approved in Japan against blood vessel obstruction, may enhance cellular autophagy.

Taken together these results show the ability of drug networks in identifying novel applications for existing drugs, as well as to charachterise novel molecules by looking at the known properties of their connected neighboring compounds.

\section{Drug-Networks based on side-effects similarity}

In addition to methods based on GEPs, other kinds of drug similarity metrics have been developed to infer drug networks.
Campillos et al [52] mined the Unified Medical Language System (UMLS) [53] ontology for medical symptoms and extracted informations about known sideeffects of a large number of marketed drugs. They then assembled a network in which two drugs were connected by an edge if known to cause similar adverse reactions. In this way, they were able to discover unexpected connections among drugs with dissimilar chemical structure and different therapeutic indication. A number of these similarities were experimentally verified and confirmed previously unreported drug-target relations. Specifically, the authors predicted, and experimentally validated with in-vitro and cell assays, that the nervous system drugs pergolide, paroxetine, and fluoxetine share a common target with rabeprazole, a proton pump inhibitor, approved for relieving duodenal ulcer symptoms and for treating ulcerative gastroesophageal reflux disease.

\section{Conclusions}

Molecular and drug networks are powerful approaches to speed up and improve both the drug discovery process and drug repositioning. We expect that their full integration into the academic and industrial drug discovery pipeline will likely have a major impact.

We have reviewed three different broad approaches used to identify drug MoA by harnessing the power of networks, at three different level of abstraction: transcriptional networks, protein networks and drug networks.

The role of transcriptional networks in determining mode of action of small molecules is undoubtebly growing, thanks to availability of more detailed gene network models and to the ever increasing amount and quality of GEPs. Indeed, thanks to the new sequencing technologies, it is now possible to achieve extreme multiplexing, i.e. mix different RNA samples in the same assay, thus enormously reducing the costs of obtaining GEPs in specific cell types and tissues. For example, a new high-throughput sequencing strategy was recently described [54], allowing measurement of gene expression profiles of hundreds of genes in thousands of samples to identify small molecules for therapy of hormone-refractory prostate cancer.

Signaling networks are the elective choice to model and identify drug Mode of Action, since most of the known effects of small molecules are mediated by kinases and phosphates. Despite the recent progress in fast and effective computational approaches, some of which were here reviewed, the major drawbacks is the experimental effort required to perform comprehensive phosphoproteomic time-course experiments. Increasing precision and decreasing costs of performing genome-wide phosphoproteomic measurements will likely lead to an increased use of signaling network models in the drug discovery.

Drug network are a unique tool to explore similarities and differences between drugs, and between drugs 
and diseases. The topology of "drug-drug" and "drugdisease" networks allows the inference of new applications for already approved drugs, and the elucidation of the MoA of small-molecules. Unlike transcriptional and signaling networks, these methods provide little or no mechanistic insights, since they rely on broad similarities in phenotypic effects of drugs, and not on detailed molecular interactions. Nevertheless, because of this, they do not require ad-hoc and expensive experiments, but can rely on the huge amount of publicly available transcriptional, chemoinformatics and literature-derived data, from which similarities and corresponding drug networks can be inferred. These data already exist and have not been fully exploited yet, and arguably it will be able to reveal a massive number of new meaningful connections.

Interestingly, at the time of writing, massive transcriptomics experiments are ongoing where thousands of cell lines are being treated with thousands of small molecules to detect gene expression changes following treatment with each compound (refer to the NIH LINCS initiative http://www.lincsproject.org). Such a trove of data can be analysed by network-based approaches to yield unprecedented insights connecting small molecules, pathways and diseases.

We have considered three different types of networks, each derived from different sources of experimental data or literature-based analysis. Each network type provides a different and potentially complementary view of the drug MoA with potential applications both in drug discovery/lead optimisation and for drug repositioning. It can be therefore expected that combining transcriptional, protein and drug networks will lead to an enhanced understanding of drug's mode of action.

In order to achieve this long-term objective, however, some outstanding challenges should be solved. For example, it is not obvious how to predict from cell-based data, such as GEPs following treatment, the effect of a compund in a tissue or in the whole-organism. Moreover, the general applicability of these approaches in the face of cellular and/or patient heterogeneity has yet to be proven. Linking models of signalling to transcriptional networks would allow to integrate proteomics and gene-expression data, but how to best achieve this is still an open question [55]. Moreover, it is still not clear what a good drug should look like in context of networks, since no established criteria are available yet.

Ultimately, quantitative models of small molecule function promise to link molecular events at cellular level to phenotypical outcomes and to cellular and body-level effects.
Authors' contributions

All authors read and approved the final manuscript.

\section{Acknowledgements}

This work was supported by a Fondazione Telethon grant to DdB (TGM11SB1) and the EU FP7 Grant BioPreDyn (ECFP7-KBBE-2011-5, Grant number 289434) $\mathrm{Ddb}$ and JSR. FI is an EMBL - EBI and Wellcome Trust Sanger Institute Post-Doctoral (ESPOD) fellow

\section{Author details}

${ }^{1}$ European Molecular Biology Laboratory - European Bioinformatics Institute, Wellcome Trust Genome Campus, Cambridge CB10 1SD, UK. ${ }^{2}$ Telethon Institute of Genetics and Medicine, Naples, Italy. ${ }^{3}$ Cancer Genome Project, Wellcome Trust Sanger Institute, Hinxton CB10 1SA, UK. ${ }^{4}$ Deptartment of Electrical Engineering and Information Technology, University of Naples "Federico II", Naples, Italy.

Received: 23 November 2012 Accepted: 19 July 2013

Published: 13 December 2013

\section{References}

1. Belcastro V, Siciliano V, Gregoretti F, Mithbaokar P, Dharmalingam G, Berlingieri S, lorio F, Oliva G, Polishchuck R, Brunetti-Pierri N, di Bernardo $D$ : Transcriptional gene network inference from a massive dataset elucidates transcriptome organization and gene function. Nucleic Acids Res 2011, 39(20):8677-8688.

2. Vidal M, Cusick M, Barabsi AL: Interactome networks and human disease. Cell 2011, 23(144):968-998.

3. Newman M: The structure and function of complex networks. SIAM Rev 2003, 45(45):167-256.

4. Barabsi AL, Oltvai ZN: Network biology: understanding the cell's functional organization. Nat Rev Genet 2004, 5(5):101-113.

5. Schadt EE: Molecular networks as sensors and drivers of common human diseases. Nature 2009, 461(7261):218-223.

6. Bansal M, Belcastro V, Ambesi-Impiombato A, di Bernardo D: How to infer gene networks from expression profiles. Mol Syst Bio/ 2007, 3:1-10.

7. Gardner TS, di Bernardo D, Lorenz D, Collins JJ: Inferring genetic networks and identifying compound mode of action via expression profiling. Science 2003, 301(5629):102-105.

8. di Bernardo D, Thompson MJ, Gardner TS, Chobot SE, Eastwood EL, Wojtovich AP, Elliott SJ, Schaus SE, Collins JJ: Chemogenomic profiling on a genome-wide scale using reverse-engineered gene networks. Nat Biotechnol 2005, 23(3):377-383.

9. Xing H, Gardner TS: The mode-of-action by network identification (MNI) algorithm: a network biology approach for molecular target identification. Nat Protocols 2006, 1(6):2551-2554.

10. Ergün A, Lawrence CA, Kohanski MA, Brennan TA, Collins JJ: A network biology approach to prostate cancer. Mol Syst Biol 2007, 3:1-6.

11. Mani KM, Lefebvre C, Wang K, Lim WK, Basso K, Dalla-Favera R, Califano A A systems biology approach to prediction of oncogenes and molecular perturbation targets in B-cell lymphomas. Mol Syst Biol 2008, 4:1-9.

12. Bansal M, Della Gatta G, di Bernardo D: Inference of gene regulatory networks and compound mode of action from time course gene expression profiles. Bioinformatics 2006, 22(7):815-822.

13. Della Gatta G, Bansal M, Ambesi-Impiombato A, Antonini D, Missero C, di Bernardo D: Direct targets of the Trp63 transcription factor revealed by a combination of gene expression profiling and reverse engineering. Genome Res 2008, 18:939-948.

14. Niola F, Xudong Z, Singh D, Castano A, Sullivan R, Lauria M, Nam HS, Zhuang $Y$, Benezra $R$, di Bernardo D, lavarone A, Lasorella A: Id proteins synchronize stemness and anchorage to the niche of neural stem cells. Nature Cell Biol 2012, 14(5):477-487.

15. Beal MJ, Falciani F, Ghahramani Z, Rangel C, Wild DL: A Bayesian approach to reconstructing genetic regulatory networks with hidden factors. Comput Appl Biosci: CABIOS 2005, 21(3):349-356.

16. Husmeier $D$ : Sensitivity and specificity of inferring genetic regulatory interactions from microarray experiments with dynamic Bayesian networks. Comput App/ Biosci: CABIOS 2003, 19(17):2271-2282. 
17. Barenco M, Tomescu D, Brewer D, Callard R, Stark J, Hubank M: Ranked prediction of p53 targets using hidden variable dynamic modeling. Genome Biol 2006, 7(3):R25.

18. Honkela A, Girardot C, Gustafson EH, Liu YH, Furlong EEM, Lawrence ND, Rattray M: Model-based method for transcription factor target identification with limited data. Proc Natl Acad Sci 2010, 107(17):7793-7798

19. Douglas SM, Montelione GT, Gerstein M: PubNet: a flexible system for visualizing literature derived networks. Genome Bio/ 2005, 6(9):R80

20. Carro M, Lim W, Alvarez M, Bollo R, Zhao X, Snyder E, Sulman E, Anne S, Doetsch F, Colman H, Lasorella A, Aldape K, Califano A, lavarone A: The transcriptional network for mesenchymal transformation of brain tumours. Nature 2010, 463(7279):318-325.

21. Kotelnikova E, Shkrob MA, Pyatnitskiy MA, Ferlini A, Daraselia N: Novel approach to meta-analysis of microarray datasets reveals muscle remodeling-related drug targets and biomarkers in duchenne muscular dystrophy. PLOS Comp Biol 2012, 8(2):e1002365.

22. Yang R, Niepel M, Mitchison TK, Sorger PK: Dissecting variability in responses to cancer chemotherapy through systems pharmacology. Clinic Pharmacol Ther 2010, 88:34-38.

23. Terfve C, Saez-Rodriguez J: Modeling signaling networks using high-throughput phospho-proteomics. Adv Exp Med Bio/ 2012 736:19-57.

24. Fitzgerald JB, Schoeberl B, Nielsen UB, Sorger PK: Systems biology and combination therapy in the quest for clinical efficacy. Nat Chem Biol 2006, 2(9):458-466.

25. Lehár J, Zimmermann GR, Krueger AS, Molnar RA, Ledell JT, Heilbut AM, Short GF, Giusti LC, Nolan GP, Magid OA, Lee MS, Borisy AA, Stockwell BR, Keith CT: Chemical combination effects predict connectivity in biological systems. Mol Syst Biol 2007, 3:80

26. Hendriks BS: Functional pathway pharmacology: chemical tools, pathway knowledge and mechanistic model-based interpretation of experimental data. Curr Opin Chem Biol 2010, 14(4):489-497.

27. Schoeberl B, Pace EA, Fitzgerald JB, Harms BD, Xu L, Nie L, Linggi B, Kalra A, Paragas V, Bukhalid R, Grantcharova V, Kohli N, West KA, Leszczyniecka M, Feldhaus MJ, Kudla AJ, Nielsen UB: Therapeutically targeting ErbB3 a key node in ligand-induced activation of the ErbB receptor-PI3K axis. Sci Signal 2009, 2(77):ra31.

28. Iadevaia S, Lu Y, Morales FC, Mills GB, Ram PT: Identification of optimal drug combinations targeting cellular networks: integrating phospho-proteomics and computational network analysis. Cancer Res 2010, 70(17):6704-6714.

29. Hendriks BS, Seidl KM, Chabot JR: Two additive mechanisms impair the differentiation of 'substrate-selective' p38 inhibitors from classical p38 inhibitors in vitro. BMC Syst Bio/ 2010, 4:23.

30. Morris MK, Saez-Rodriguez J, Sorger PK, Lauffenburger DA: Logic-based models for the analysis of cell signaling networks. Biochem 2010, 49(15):3216-3224.

31. Watterson S, Marshall S, Ghazal P: Logic models of pathway biology. Drug Discov Today 2008, 13(9-10):447-456.

32. Wang RS, Saadatpour A, Albert R: Boolean modeling in systems biology an overview of methodology and applications. Phys Biol 2012, 9(5):055001

33. Saez-Rodriguez J, Alexopoulos LG, Zhang M, Morris MK, Lauffenburger DA, Sorger PK: Comparing signaling networks between normal and transformed Hepatocytes using discrete logical models. Cancer Res 2011

34. Mitsos A, Melas I, Siminelakis P, Chairakaki A, Saez-Rodriguez J, Alexopoulos $L G$ : Identifying drug effects via pathway alterations using an integer linear programming optimization formulation on phosphoproteomic data. PLoS Comp Biol 2009, 5(12):e1000591.

35. Saadatpour A, Wang RS, Liao A, Liu X, Loughran TP, Albert l, Albert R Dynamical and structural analysis of a $T$ cell survival network identifies novel candidate therapeutic targets for large granular lymphocyte Leukemia. PLoS Comput Biol 2011, 7(11):e1002267.

36. Sahin O, Fröhlich H, Löbke C, Korf U, Burmester S, Majety M, Mattern J, Schupp I, Chaouiya C, Thieffry D, Poustka A, Wiemann S, Beissbarth T, Arlt D: Modeling ERBB receptor-regulated G1/S transition to find novel targets for de novo trastuzumab resistance. BMC Syst Biol 2009, 3:1.
37. Iskar M, Campillos M, Kuhn M, Jensen L, van Noort V, Bork P. Drug-induced regulation of target expression. PLoS Comput Bio/ 2010, 6(9):1929-1935.

38. Iorio F, Rittman T, Ge H, Menden M, Saez-Rodriguez J: Transcriptional data: a new gateway to drug repositioning? Drug Discov Today 2013, 18(7-8):350-357.

39. Iorio F, Isacchi A, di Bernardo D: Identification of small molecules enhancing autophagic function from drug network analysis. Autophagy 2010, 6(8):1204-1205

40. Dudley JT, Deshpande T, Butte AJ: Exploiting drug-disease relationships for computational drug repositioning. Brief Bioinformatics 2011, 12(4):303-311.

41. Dudley JT, Sirota M, Shenoy M, Pai RK, Roedder S, Chiang AP, Morgan AA Sarwal MM, Pasricha PJ, Butte AJ: Computational repositioning of the anticonvulsant topiramate for inflammatory bowel disease. $\mathrm{SCl}$ Trans/ Med 2011, 3(96):96ra76-96ra76.

42. Sirota M, Dudley JT, Kim J, Chiang AP, Morgan AA, Sweet-Cordero A, Sage J, Butte AJ: Discovery and preclinical validation of drug indications using compendia of public gene expression data. Sci Trans/ Med 2011, 3(96):96ra77-96ra77.

43. Kunkel SD, Suneja M, Ebert SM, Bongers KS, Fox DK, Malmberg SE, Alipour F, Shields RK, Adams CM: mRNA Expression signatures of human skeletal muscle atrophy identify a natural compound that increases muscle mass. Cell Metabolism 2011, 13(6):627-638.

44. Shigemizu D, Hu Z, Hung JH, Huang CL, Wang Y, DeLisi C: Using functional signatures to identify repositioned drugs for breast, myelogenous Leukemia and prostate cancer. PLOS Comput Biol 2012 8(2):e1002347.

45. Iorio F, Bosotti R, Scacheri E, Belcastro V, Mithbaokar P, Ferriero R, Murino $L$, Tagliaferri R, Brunetti-Pierri N, Isacchi A: Discovery of drug mode of action and drug repositioning from transcriptional responses. Proc Natl Acad Sci 2010, 107(33):14621.

46. Ma C, Chen H, Huang Y, Chen Y: Constructing a compound mode-ofaction network for personalized drug effectiveness prediction. Proc First ACM Intl Conf Bioinformatics Comput Biol 2010:520-528.

47. Lamb J, Crawford E, Peck D, Modell J, Blat I, Wrobel M, Lerner J, Brunet J, Subramanian A, Ross K: The Connectivity Map: using gene-expression signatures to connect small molecules, genes, and disease. Science (New York, NY) 1929, 313(5795).

48. Subramanian A, Tamayo P, Mootha V, Mukherjee S, Ebert B, Gillette M, Paulovich A, Pomeroy S, Golub T, Lander E: Gene set enrichment analysis: a knowledge-based approach for interpreting genome-wide expression profiles. Proc Natl Acad Sci USA 2005, 102(43):15545.

49. Lamb J: The Connectivity Map: a new tool for biomedical research. Nat Rev Cancer 2007, 7:54-60.

50. $\mathrm{Hu} \mathrm{G}$, Agarwal P: Human disease-drug network based on genomic expression profiles. PLOS ONE 2009, 4(8):e6536.

51. Barrett T: NCBI GEO: mining millions of expression profiles-database and tools. Nucleic Acids Res 2004, 33(Database issue):D562-D566.

52. Campillos M, Kuhn M, Gavin AC, Jensen LJ, Bork P: Drug target identification using side-effect similarity. Science 2008 321(5886):263-266.

53. Bodenreider O: The Unified Medical Language System (UMLS): integrating biomedical terminology. Nucleic Acids Res 2004, 32(90001):267D-270D.

54. Li H, Zhou H, Wang D, Qiu J, Zhou Y, Li X, Rosenfeld MG, Ding S, Fu XD: Versatile pathway-centric approach based on high-throughput sequencing to anticancer drug discovery. Proc Natl Acad Sci 2012, 109(12):4609-4614

55. lyengar R, Zhao S, Chung SW, Mager DE, Gallo JM: Merging systems biology with pharmacodynamics. SciTrans/ Med 2012, 4(126):126ps7.

doi:10.1186/1752-0509-7-139

Cite this article as: lorio et al:: Network based elucidation of drug response:

from modulators to targets. BMC Systems Biology 2013 7:139. 